\title{
Rheumatoid arthritis-associated autoantibodies and subclinical interstitial lung disease: the Multi-Ethnic Study of Atherosclerosis
}

\author{
Elana J Bernstein, ${ }^{1}$ R Graham Barr, ${ }^{1,2}$ John H M Austin, ${ }^{3}$ Steven M Kawut, ${ }^{4}$ \\ Ganesh Raghu, ${ }^{5}$ Jessica L Sell, ${ }^{1}$ Eric A Hoffman, ${ }^{6}$ John D Newell Jr, ${ }^{6}$ \\ Jubal R Watts $\mathrm{Jr}^{7}{ }^{7}$ P Hrudaya Nath, ${ }^{7}$ Sushil K Sonavane, ${ }^{7}$ Joan M Bathon, ${ }^{1}$ \\ Darcy S Majka, ${ }^{8}$ David J Lederer ${ }^{1,2}$
}

- Additional material is published online only. To view please visit the journal online (http://dx.doi.org/10.1136/ thoraxjn-2016-208932)

For numbered affiliations see end of article.

\section{Correspondence to}

Dr David J Lederer,

Departments of Medicine and Epidemiology (in Pediatrics), Columbia University Medical Center, 161 Fort Washington Ave, Room 3-321A, New York, NY 10032, USA:

davidlederer@columbia.edu

DSM and DJL contributed equally.

Received 18 May 2016 Revised 21 July 2016 Accepted 27 July 2016 Published Online First 8 September 2016

\section{CrossMark}

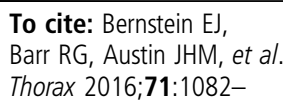

\section{ABSTRACT}

Background Adults with interstitial lung disease (ILD) often have serologic evidence of autoimmunity of uncertain significance without overt autoimmune disease. We examined associations of rheumatoid arthritis (RA)-associated antibodies with subclinical ILD in community-dwelling adults.

Methods We measured serum rheumatoid factor (RF) and anticyclic citrullinated peptide antibody (anti-CCP) and high attenuation areas (HAAs; CT attenuation values between -600 and -250 Hounsfield units) on cardiac CT in 6736 community-dwelling US adults enrolled in the Multi-Ethnic Study of Atherosclerosis. We measured interstitial lung abnormalities (ILAs) in 2907 full-lung CTs at 9.5-year median follow-up. We used generalised linear and additive models to examine associations between autoantibodies and both HAA and ILA, and tested for effect modification by smoking.

Results In adjusted models, HAA increased by $0.49 \%$ (95\% $\mathrm{Cl} 0.11 \%$ to $0.86 \%$ ) per doubling of RF IgM and by $0.95 \%(95 \% \mathrm{Cl} 0.50 \%$ to $1.40 \%)$ per RF IgA doubling. ILA prevalence increased by $11 \%(95 \% \mathrm{Cl} 3 \%$ to $20 \%$ ) per RF IgA doubling. Smoking modified the associations of both RF IgM and anti-CCP with both HAA and ILA (interaction $p$ values varied from 0.01 to 0.09 ). Among ever smokers, HAA increased by $0.81 \%$ (95\% Cl $0.33 \%$ to $1.30 \%)$ and ILA prevalence increased by $14 \%(95 \% \mathrm{Cl} \%$ to $24 \%$,) per RF IgM doubling; and HAA increased by $1.31 \%(95 \% \mathrm{Cl} 0.45 \%$ to $2.18 \%)$ and ILA prevalence increased by $13 \%(95 \% \mathrm{Cl}$ $2 \%$ to $24 \%$ ) per anti-CCP doubling. Among never smokers, no meaningful associations were detected. Conclusions RA-related autoimmunity is associated with both quantitative and qualitative subclinical ILD phenotypes on $\mathrm{CT}$, particularly among ever smokers.

\section{INTRODUCTION}

The pulmonary parenchyma is a frequent target of autoimmunity. Approximately 30\% of adults diagnosed with interstitial lung disease (ILD) have an underlying established autoimmune disease, such as rheumatoid arthritis (RA), systemic sclerosis, Sjögren's syndrome, mixed connective tissue disease or an inflammatory myopathy. ${ }^{1}$ Indeed, ILD may be the initial or sole clinical manifestation of an autoimmune disease, and those diagnosed with

\section{Key messages}

What is the key question?

- Is rheumatoid arthritis (RA)-associated autoimmunity associated with subclinical interstitial lung disease (ILD)?

\section{What is the bottom line?}

- We provide evidence that higher levels of RA-associated autoantibodies - even in the normal range - are associated with both quantitative and qualitative CT phenotypes of subclinical ILD in community-dwelling adults, particularly among ever smokers.

\section{Why read on?}

- This study provides the first evidence linking autoimmunity to subclinical lung injury, inflammation and extracellular matrix remodelling. These data suggest that clinical trials to prevent ILD in at-risk adults could focus on targeting autoimmunity.

idiopathic non-specific interstitial pneumonia often have autoimmune features without an established autoimmune disease..$^{2-5}$

Subclinical ILD on CT is surprisingly common in at-risk groups, including cigarette smokers, relatives of those with familial pulmonary fibrosis, and those with established autoimmune diseases. ${ }^{6-8}$ For example, $8 \%$ of adult smokers and $33 \%$ of adults with RA without pulmonary symptoms have subclinical ILD. ${ }^{7} 8$ Examination of subclinical ILD holds the promise of identifying biological pathways that contribute to early alveolar epithelial cell injury and extracellular matrix remodelling prior to the onset of established fibrosis. Insights into these early processes may lead to the identification of novel preventative and therapeutic targets. High attenuation areas (HAAs), a novel quantitative measure of lung attenuation, have strong construct validity as a biomarker of subclinical lung inflammation and extracellular matrix remodelling. ${ }^{9}$ HAA is associated with reduced FVC, reduced exercise capacity, elevated serum levels of matrix metalloproteinase-7 and 
interleukin-6, and a higher risk of interstitial lung abnormalities (ILAs) on CT and a higher all-cause mortality rate. ${ }^{9}$

The availability of population-based cohorts affords the opportunity to examine risk factors for subclinical ILD on CT. Based on the strong links between autoimmunity and ILD, we aimed to determine whether serum markers of autoimmunityin this case, serum rheumatoid factor (RF) $\operatorname{IgM}$ and $\operatorname{IgA}$ and anticyclic citrullinated peptide antibody (anti-CCP)—were associated with HAA and ILA in a population-based cohort. We hypothesised that baseline levels of RF IgM, RF IgA and anti-CCP would be associated with a greater percentage of HAA on baseline CT in a cross-sectional analysis, and with the presence of ILA on chest CT performed a decade later.

\section{METHODS}

\section{Participants and data sources}

The Multi-Ethnic Study of Atherosclerosis (MESA) is a multicentre, prospective cohort study of 6814 men and women who were between 45 and 84 years of age when they were recruited in 2000-2002 (Exam 1). ${ }^{10}$ Participants were recruited from six sites in the USA: Forsyth County, North Carolina; northern Manhattan and the Bronx, New York; Baltimore City and Baltimore County, Maryland; St. Paul, Minnesota; Chicago, Illinois; and Los Angeles, California. MESA exclusion criteria included clinical cardiovascular disease, weight $>136 \mathrm{~kg}$, chest CT imaging during the year prior to enrolment, or any barrier to long-term participation. Participants returned for four subsequent visits, the most recent occurring in 2010-2012 (Exam 5). See the online supplementary data for additional details.

\section{Measurement of exposure and covariates}

RF and second-generation anti-CCP were measured in frozen serum samples collected at Exam 1. Isotype-specific IgM and IgA RF and IgG anti-CCP were quantified by ELISA using commercial kits (TheraTest Labs, Lombard, Illinois, USA). ${ }^{11}$ Testing was performed at TheraTest Labs. Intra-assay coefficients of variation for RF IgM, RF IgA and anti-CCP were $<10 \%$. $^{11}$ The levels of RF IgM, RF IgA and anti-CCP were expressed in $\mathrm{U} / \mathrm{mL}$.

Demographics, educational attainment and tobacco use data were collected by questionnaires administered at Exam 1. Height, weight and waist circumference were measured during Exam 1. ${ }^{10}$ Detailed information about MESA has been previously published. ${ }^{10} 1213$ See the online supplementary data for additional details.

\section{Quantitative CT attenuation on cardiac CT scan}

Cardiac CT scans were performed on 6812 MESA participants at Exam 1 (2000-2002), using multidetector and electron beam scanners (three sites each) through a standardised protocol. ${ }^{14}$ Image attenuation was assessed using a modified version of the Pulmonary Analysis Software Suite at a single reading centre by trained readers without knowledge of participants' autoantibody levels or other characteristics. ${ }^{15} 16$ HAAs are defined as the volume of lung having a CT attenuation value between -600 and -250 Hounsfield units, a range that captures ground glass and reticular abnormalities but excludes areas of higher density such as blood vessels, nodules and consolidations. ${ }^{12}$ Per cent emphysema was defined as the percentage of lung voxels less than -950 Hounsfield units. ${ }^{13}$ Numerous previous studies have used MESA cardiac CT scans to examine pulmonary emphysema. $^{13} 17$

\section{Interstitial lung abnormalities on chest CT}

Two-thousand nine-hundred seven full lung CT scans performed at Exam 5 (2010-2012) were each visually inspected by one expert radiologist for the presence or absence of the following ILAs involving $>5 \%$ of lung zone in a non-dependent fashion: ground glass abnormalities, reticular abnormalities, diffuse centrilobular nodularity, nonemphysematous cysts, honeycombing and traction bronchiectasis. ${ }^{7} 1819$ Evidence of bilateral fibrosis in multiple lobes in a subpleural distribution associated with honeycombing and traction bronchiectasis was also included in the definition of ILA. ${ }^{19}$

\section{Statistical analysis}

We examined the linearity of the associations between each autoantibody and HAA and ILA using generalised additive models with loess smoothing functions for continuous variables. In multivariable models, we examined autoantibody levels expressed both as continuous variables and in autoantibody quartiles. We used multiple linear regression to examine the associations between each natural log-transformed autoantibody (or autoantibody quartile) and natural log-transformed HAA, controlling for age, sex, race, study site, education, body mass index (BMI), height, waist circumference, pack-years of smoking, current smoking status, total volume of imaged lung, per cent emphysema and tube current. To ease interpretation of our $\beta$ coefficients of natural log-transformed autoantibody levels, we have presented base 2 exponentiated $\beta$ coefficients, which are the per cent differences in HAA per doubling of each autoantibody level. We also presented inverse natural $\log \beta$ coefficients for each autoantibody quartile, which are the per cent differences in HAA for each quartile compared with the lowest quartile. We estimated prevalence ratios (PRs) for the associations between each $\log _{2}$ transformed autoantibody (or autoantibody quartile) and ILA using Poisson regression with robust SE estimation, controlling for age, sex, race, pack-years of smoking and current smoking status. We have presented inverse natural $\log \beta$ coefficients from these models, which are the PRs for ILA per doubling of autoantibody level. The inverse natural $\log \beta$ coefficients for each autoantibody quartile are the PRs for ILA for each quartile compared with the lowest quartile. To examine consistency of effect, we performed analyses stratified by age, sex, race, smoking status, BMI and hepatitis $\mathrm{C}$ status at Exam 1. We used likelihood ratio tests to test for effect modification. We used multiple imputation by chained equations to account for missing covariate data. ${ }^{20}$ Analyses were performed in STATA, V.13.1 (College Station, Texas, USA) and R, V.3.1.2 (R Foundation for Statistical Computing, Vienna, Austria). See the online supplementary data for additional details.

\section{RESULTS}

Among the 6812 MESA participants who underwent cardiac CT scan at Exam 1, RF IgM and RF IgA were measured in 6736 and anti-CCP was measured in 6728 . Of the 2430 participants with non-equivocal measurements of ILA at Exam 5, RF IgM and RF IgA were measured in 2403 at Exam 1 and anti-CCP was measured in 2399. In the overall cohort, the median (IQR) RF IgM level was $8 \mathrm{U} / \mathrm{mL}(4-17 \mathrm{U} / \mathrm{mL})$, the median (IQR) RF IgA level was $9 \mathrm{U} / \mathrm{mL}(6-16 \mathrm{U} / \mathrm{mL})$ and the median (IQR) anti-CCP level was $0.2 \mathrm{U} / \mathrm{mL} \quad(0.1-0.5 \mathrm{U} / \mathrm{mL})$. The median (IQR) HAA was $121.1 \mathrm{~cm}^{3}\left(101.7-145.5 \mathrm{~cm}^{3}\right)$ and $12.6 \% \mathrm{had}$ ILA.

Baseline participant characteristics stratified by RF IgM level are shown in table 1 . The median (IQR) age was 62 (53-70) 
Table 1 Baseline characteristics by lgM rheumatoid factor quartile

\begin{tabular}{|c|c|c|c|c|}
\hline & \multicolumn{4}{|c|}{ IgM rheumatoid factor quartile } \\
\hline & $<4.03 \mathrm{U} / \mathrm{mL}$ & $4.03-8 \mathrm{U} / \mathrm{mL}$ & $8.02-17 \mathrm{U} / \mathrm{mL}$ & $>17 \mathrm{U} / \mathrm{mL}$ \\
\hline No. of subjects & 1814 & 1710 & 1616 & 1596 \\
\hline \multicolumn{5}{|l|}{ Demographics and anthropometrics } \\
\hline Age, years & $63(54-71)$ & $61(53-69)$ & $62(53-70)$ & $63(54-71)$ \\
\hline Female sex & $918(50.6)$ & $887(51.9)$ & $882(54.6)$ & $876(54.9)$ \\
\hline \multicolumn{5}{|l|}{ Race/ethnicity } \\
\hline White & $694(38.3)$ & $727(42.5)$ & $635(39.3)$ & $543(34.0)$ \\
\hline African-American & $450(24.8)$ & $398(23.3)$ & $443(27.4)$ & $559(35.0)$ \\
\hline Chinese-American & $291(16.0)$ & $207(12.1)$ & $162(10.0)$ & $143(9.0)$ \\
\hline Hispanic & $379(20.9)$ & $378(22.1)$ & $376(23.3)$ & $351(22.0)$ \\
\hline Height, $\mathrm{cm}$ & $166.0 \pm 9.9$ & $166.7 \pm 10.0$ & $166.5 \pm 10.1$ & $166.2 \pm 10.2$ \\
\hline Weight, Ibs & $171.0 \pm 37.1$ & $173.2 \pm 39.3$ & $174.8 \pm 39.2$ & $174.4 \pm 37.2$ \\
\hline Body mass index, $\mathrm{kg} / \mathrm{m}^{2}$ & $28.1 \pm 5.3$ & $28.2 \pm 5.4$ & $28.5 \pm 5.6$ & $28.6 \pm 5.5$ \\
\hline \multicolumn{5}{|l|}{ Body mass index category, $\mathrm{kg} / \mathrm{m}^{2}$} \\
\hline Underweight & $16(0.9)$ & $12(0.7)$ & $15(0.9)$ & $13(0.8)$ \\
\hline Normal & $538(29.7)$ & $508(29.7)$ & $432(26.7)$ & $401(25.1)$ \\
\hline Overweight & $702(38.7)$ & $647(37.8)$ & $644(39.9)$ & $645(40.4)$ \\
\hline Obese & $558(30.8)$ & $543(31.8)$ & $525(32.5)$ & $537(33.7)$ \\
\hline Waist circumference, $\mathrm{cm}$ & $97.5 \pm 13.9$ & $98.0 \pm 14.9$ & $98.5 \pm 14.5$ & $98.6 \pm 14.3$ \\
\hline Hip circumference, $\mathrm{cm}$ & $104.9 \pm 11.4$ & $105.4 \pm 11.4$ & $106.1 \pm 11.5$ & $106.0 \pm 11.5$ \\
\hline \multicolumn{5}{|l|}{ Smoking } \\
\hline Never smoker & $854(47.1)$ & $807(47.2)$ & $727(45.0)$ & $663(41.6)$ \\
\hline Former smoker & $735(40.5)$ & $658(38.5)$ & $655(40.6)$ & $683(42.8)$ \\
\hline Current smoker & $224(12.4)$ & $245(14.3)$ & $232(14.4)$ & $249(15.6)$ \\
\hline Cigarette pack-years (among ever smokers) & $13.8(3.0-30.0)$ & $14.0(3.3-33.0)$ & $15.0(3.5-34.0)$ & $15.0(3.4-33.4)$ \\
\hline \multicolumn{5}{|l|}{ Spirometry } \\
\hline $\mathrm{FEV}_{1}, \mathrm{~L}$ & $2.4 \pm 0.7$ & $2.4 \pm 0.7$ & $2.4 \pm 0.7$ & $2.3 \pm 0.7$ \\
\hline $\mathrm{FEV}_{1}, \%$ predicted & $94.9 \pm 17.9$ & $92.7 \pm 17.4$ & $94.1 \pm 18.2$ & $93.4 \pm 19.0$ \\
\hline FVC, L & $3.2 \pm 0.9$ & $3.2 \pm 1.0$ & $3.2 \pm 1.0$ & $3.1 \pm 0.9$ \\
\hline FVC, \%predicted & $95.8 \pm 16.1$ & $94.7 \pm 15.9$ & $95.3 \pm 16.1$ & $95.8 \pm 17.2$ \\
\hline $\mathrm{FEV}_{1} / \mathrm{FVC}$ ratio & $0.75 \pm 0.08$ & $0.75 \pm 0.08$ & $0.75 \pm 0.08$ & $0.74 \pm 0.09$ \\
\hline \multicolumn{5}{|l|}{ CT } \\
\hline Total imaged lung volume (gas+tissue), $\mathrm{cm}^{3}$ & $2722.8 \pm 799.4$ & $2794.0 \pm 764.4$ & $2789.8 \pm 864.0$ & $2772.8 \pm 788.7$ \\
\hline Emphysema, \% & $2.8(1.2-5.5)$ & $3.0(1.3-5.8)$ & $2.8(1.2-5.8)$ & $2.9(1.3-5.6)$ \\
\hline \multicolumn{5}{|l|}{ Respiratory diseases } \\
\hline Self-reported emphysema, \% & $29(1.6)$ & $23(1.4)$ & $31(1.9)$ & $19(1.2)$ \\
\hline Self-reported asthma, \% & $170(9.4)$ & $173(10.1)$ & $154(9.5)$ & $164(10.3)$ \\
\hline Hepatitis C & $8(0.4)$ & $4(0.2)$ & $7(0.4)$ & $28(1.8)$ \\
\hline
\end{tabular}

years. Fifty-three per cent were female, $38.6 \%$ self-identified as white, $27.5 \%$ as African-American, $11.9 \%$ as Chinese-American and $22.0 \%$ as Hispanic. Fourteen per cent were current smokers, whereas $45.3 \%$ were never smokers. The median (IQR) number of pack-years among ever smokers was 14.5 (3.3-33.0). Most baseline characteristics were similar across quartiles of RF IgM, RF IgA and anti-CCP levels (table 1, see online supplementary tables S1 and S2), with the exceptions of race, smoking status and hepatitis $\mathrm{C}$ seropositivity. AfricanAmerican race was more prevalent among those with higher RF IgM, RF IgA or anti-CCP levels. Current smoking status and hepatitis $\mathrm{C}$ seropositivity were more prevalent among those with higher RF IgM or RF IgA levels.

\section{IgM rheumatoid factor, HAA and ILA}

HAA increased across RF IgM quartiles ( $p$ value for trend $=0.03$; table 2 ). In an unadjusted model, HAA increased by $0.60 \%$ per doubling of RF IgM (95\% CI $0.13 \%$ to $1.07 \%$, $\mathrm{p}$ value $=0.01$; table 2 ). In a fully adjusted model, the $\mathrm{p}$ value for the interaction between smoking status and RF IgM was 0.09 (table 2). In fully adjusted models, among ever smokers, HAA increased by $0.81 \% \quad(95 \%$ CI $0.33 \%$ to $1.30 \%$, $\mathrm{p}$ value $=0.001)$ per doubling of RF IgM, but increased by only $0.15 \%(95 \% \mathrm{CI}-0.44 \%$ to $0.75 \%$, p value $=0.62)$ per doubling of RF IgM among never smokers (table 2 and figure 1A).

The prevalence of ILA increased from $12.0 \%$ among those with an RF IgM level $<4.03 \mathrm{U} / \mathrm{mL}$ (quartile 1) to $16.0 \%$ among those with an RF IgM level $>17 \mathrm{U} / \mathrm{mL}$ (quartile 4; p value for trend $=0.007$; table 2 ). In an unadjusted model, the prevalence of ILA increased by 10\% per doubling of RF IgM (PR 1.10, $95 \%$ CI 1.02 to 1.17 , p value $=0.009$ ). In a fully adjusted model, the $\mathrm{p}$ value for the interaction between smoking status and RF IgM was 0.04 (table 2). In fully adjusted models, among ever smokers, the prevalence of ILA increased by $14 \%$ per doubling of RF $\operatorname{IgM}$ (PR 1.14, 95\% CI 1.05 to 1.24, $\mathrm{p}$ value $=0.002$ ), while among never smokers, there was no 


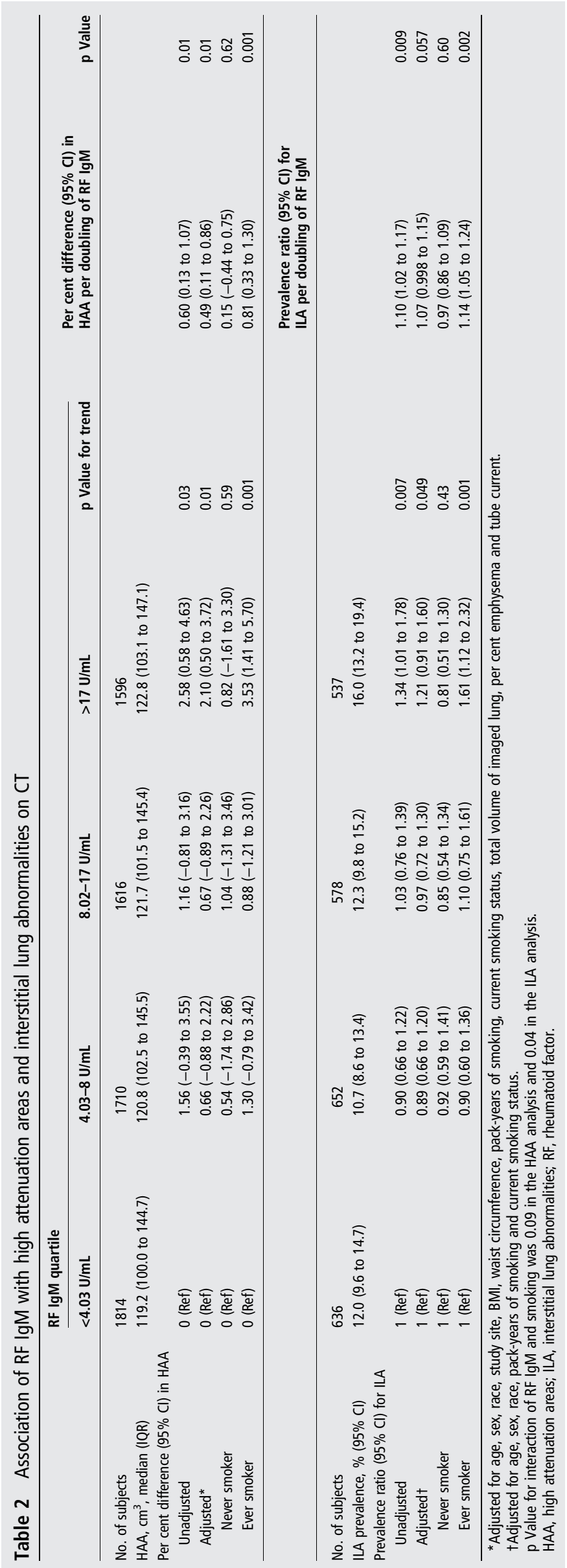

statistically significant association between RF IgM and the prevalence of ILA (PR $0.97,95 \%$ CI 0.86 to 1.09 , p value $=0.60$; table 2 and figure $1 \mathrm{D})$.

\section{IgA rheumatoid factor, HAA and ILA}

HAA increased across RF IgA quartiles ( $p$ value for trend $<0.001$; table 3 ). In a fully adjusted model, HAA increased by $0.95 \%$ per doubling of RF IgA (95\% CI $0.50 \%$ to $1.40 \%$, $\mathrm{p}$ value $<0.001$; table 3 ). In a fully adjusted model, the $\mathrm{p}$ value for the interaction between smoking status and RF IgA was 0.15 (table 3). In fully adjusted models, among ever smokers, HAA increased by $1.35 \%$ (95\% CI $0.78 \%$ to $1.92 \%$, p value $<0.001$ ) per doubling of RF IgA, and increased by $0.55 \%(95 \% \mathrm{CI}$ $-0.17 \%$ to $1.29 \%$, $\mathrm{p}$ value $=0.14$ ) per doubling of $\mathrm{RF} \operatorname{IgA}$ among never smokers (table 3 and figure $1 \mathrm{~B}$ ).

The prevalence of ILA increased from 9.6\% among those with an RF IgA level $<6.04 \mathrm{U} / \mathrm{mL}$ (quartile 1) to $16.1 \%$ among those with an RF IgA level $>15.86 \mathrm{U} / \mathrm{mL}$ (quartile 4; $\mathrm{p}$ value for trend $=0.001$; table 3 ). In a fully adjusted model, the prevalence of ILA increased by $11 \%$ per doubling of RF IgA (PR $1.11,95 \%$ CI 1.03 to $1.20, \mathrm{p}$ value $=0.006$ ). The $\mathrm{p}$ value for the interaction between smoking status and RF IgA was 0.31 . In fully adjusted models, among ever smokers, the prevalence of ILA increased by $14 \%$ per doubling of RF IgA (PR 1.14, 95\% CI 1.05 to 1.23 , $\mathrm{p}$ value $=0.001$ ), while among never smokers, there was no statistically significant association between RF IgA and the prevalence of ILA (PR 1.04, 95\% CI 0.88 to 1.23, $\mathrm{p}$ value $=0.68)($ table 3 and figure $1 \mathrm{E}$ ).

\section{Anti-CCP antibody, HAA and ILA}

HAA did not increase across quartiles of anti-CCP ( $p$ value for trend $=0.42$ ). In a fully adjusted model, the $p$ value for the interaction between smoking status and anti-CCP was 0.02. In fully adjusted models, HAA increased by $1.31 \%$ (95\% CI $0.45 \%$ to $2.18 \%$, p value $=0.003$ ) per doubling of anti-CCP among ever smokers, while there was no statistically significant association between anti-CCP and HAA among never smokers $(0.40 \%$ decrease per doubling of anti-CCP, 95\% CI $-1.55 \%$ to $0.77 \%$, $\mathrm{p}$ value $=0.50$ ) (table 4 and figure $1 \mathrm{C})$.

The prevalence of ILA did not increase across quartiles of anti-CCP ( $p$ value for trend $=0.70$; table 4 ), and in an unadjusted model, there was no meaningful association between anti-CCP levels and ILA prevalence (PR 1.08, 95\% CI 0.95 to 1.23 , $\mathrm{p}$ value $=0.25$ ). In a fully adjusted model, the $\mathrm{p}$ value for the interaction between smoking status and anti-CCP was 0.01 (table 4). In fully adjusted models, among ever smokers, the prevalence of ILA increased by $13 \%$ per doubling of anti-CCP $(\mathrm{PR} 1.13,95 \%$ CI 1.02 to 1.24 , p value $=0.02)$, while among never smokers, there was no statistically significant association between anti-CCP and the prevalence of ILA (PR 0.66, 95\% CI 0.37 to 1.19 , p value $=0.17$ ) (table 4 and figure $1 \mathrm{~F})$.

Online supplementary table S3 shows the p values for the linearity of the continuous associations between autoantibody concentrations and HAA and ILA.

\section{Race-stratified analyses}

Race appeared to modify the association of anti-CCP with subclinical ILD among ever smokers. In fully adjusted models, among Hispanics, HAA increased by $3.85 \%$ (95\% CI $1.57 \%$ to $6.18 \%, \mathrm{p}$ value $=0.001$ ) per doubling of anti-CCP compared with $0.58 \%$ (95\% CI $-0.32 \%$ to $1.48 \%$, p value $=0.21$ ) for other races ( $\mathrm{p}$ value for interaction 0.004 ). See online supplementary tables S4-6 for the results of additional stratified analyses among ever smokers. 

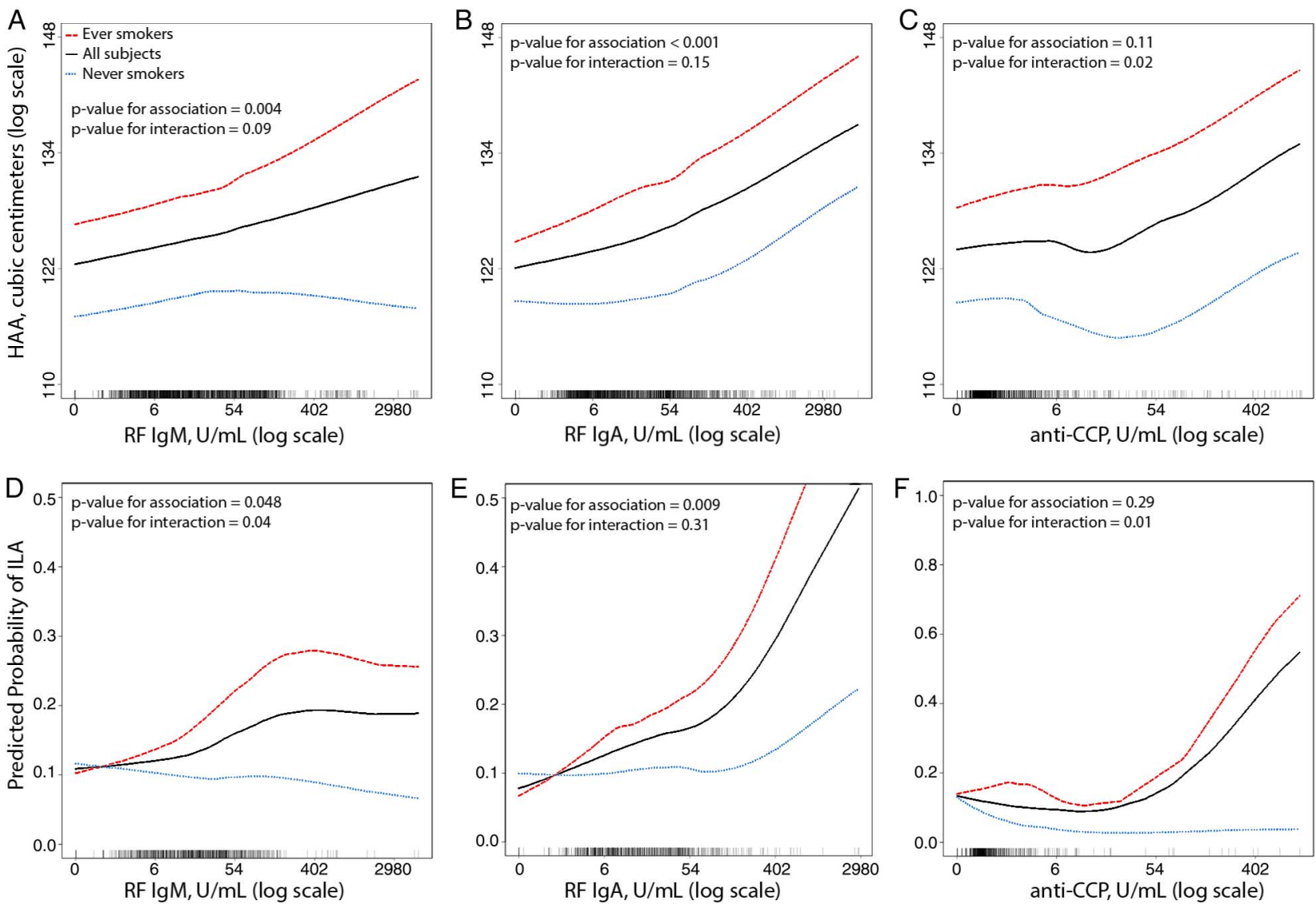

Figure 1 Continuous relationships of rheumatoid factor (RF) $\lg M(A$ and $D), R F \lg A$ ( $B$ and $E$ ) and anti-cyclic citrullinated peptide antibody (anti-CCP) ( $C$ and F) with predicted (adjusted) high attenuation area volume ( $A-C)$ and the predicted probability of interstitial lung abnormalities (ILAs) (D-F). (A-C) Smoothed regression lines (solid line: all subjects, dashed line: ever smokers, dotted line: never smokers) are adjusted for age, sex, race, study site, BMI, total volume of imaged lung, per cent emphysema and tube current. The solid line is also adjusted for pack-years of smoking and current smoking status. (D-F) Smoothed regression lines (solid line: all subjects, dashed line: ever smokers, dotted line: never smokers) are adjusted for age, sex and race. The solid line is also adjusted for pack-years of smoking and current smoking status. Each vertical tick mark on the rug plot running along the internal border of the $x$-axis represents one study participant. $p$ Values shown are for the multivariable-adjusted associations between each autoantibody and high attenuation areas (HAAs) or ILA in the overall cohort and for interactions between each autoantibody and ever smoking status.

\section{Autoantibodies as modifiers of the relationship between smoking, HAA and ILA}

Since we identified an interaction between RA-related autoantibodies and smoking status, we also examined the extent to which autoantibody levels modified the known association between smoking status and ILD. ${ }^{12}{ }^{21}$ In fully adjusted models, compared with never smoking status, ever smoking status was associated with a 2.2-fold increased prevalence of ILA (PR 2.17, 95\% CI 1.40 to 3.38 ) among participants with RF IgM levels $>17 \mathrm{U} / \mathrm{mL}$ (quartile 4) but only a $19 \%$ increased prevalence of ILA (PR 1.19, 95\% CI 0.80 to 1.79) among participants with $\mathrm{RF}$ IgM levels $<4.03 \mathrm{U} / \mathrm{mL}$ (quartile 1; p value for interaction $=0.04$; figure 2 ).

In fully adjusted models, compared with never smoking status, ever smoking status was associated with a $3.22 \%$ mean increase in HAA (95\% CI $0.79 \%$ to $5.71 \%$ ) among participants with anti-CCP levels $>0.5 \mathrm{U} / \mathrm{mL}$ (quartile 4) but only a $1.37 \%$ mean increase in HAA $(95 \% \mathrm{CI}-0.86 \%$ to $3.65 \%$ ) among participants with anti-CCP levels $<0.17 \mathrm{U} / \mathrm{mL}$ (quartile 1 ; p value for interaction $=0.02$; figure 2 ). In fully adjusted models, compared with never smoking status, ever smoking status was associated with a 2.2-fold increased prevalence of ILA (PR 2.22, 95\% CI 1.42 to 3.42) among participants with anti-CCP levels $>0.5 \mathrm{U} / \mathrm{mL}$ (quartile 4) and an $11 \%$ increased prevalence of ILA (PR 1.11, 95\% CI 0.75 to 1.63) among participants with anti-CCP levels $<0.17 \mathrm{U} / \mathrm{mL}$ (quartile $1 ; \mathrm{p}$ value for interaction $=0.01$; figure 2 ).

\section{DISCUSSION}

In this large community-based multiethnic cohort, we found that higher serum levels of both RF IgM and RF IgA were associated with HAA, a CT biomarker of subclinical lung injury and inflammation, and with ILA, a validated measure of subclinical ILD. Moreover, smoking status modified the associations of both RF IgM and anti-CCP with both HAA and ILA, with stronger associations among ever smokers compared with never smokers. These results also indicate that higher serum levels of RF IgM and anti-CCP strengthen the known association between cigarette smoking and subclinical ILD. Our findings suggest that either RA-related autoimmunity contributes to subclinical ILD among smokers without an established autoimmune disease, or, as has been previously hypothesised, that smokinginduced subclinical lung injury may lead to the local production of RA-related autoantibodies. ${ }^{22}$

We are unaware of previous studies that have examined the role of autoimmunity in subclinical ILD among communitydwelling adults. Our work builds on the results of previous studies that have identified an increased prevalence of subclinical ILD in adults with clinically diagnosed RA. ${ }^{23}{ }^{24}$ Notably, adults in our study were sampled without regard to the presence or 
Table 3 Association of RF IgA with high attenuation areas and interstitial lung abnormalities on CT

\begin{tabular}{|c|c|c|c|c|c|c|c|}
\hline & \multicolumn{5}{|l|}{$\mathrm{RF} \operatorname{IgA}$ quartile } & \multirow{2}{*}{$\begin{array}{l}\text { Per cent difference }(95 \% \mathrm{Cl}) \text { in } \\
\text { HAA per doubling of } \mathrm{RF} \text { IgA }\end{array}$} & \multirow[b]{2}{*}{$\mathrm{p}$ Value } \\
\hline & $<6.04 \mathrm{U} / \mathrm{mL}$ & $6.04-9 \mathrm{U} / \mathrm{mL}$ & $9.04-15.86 \mathrm{U} / \mathrm{mL}$ & $>15.86 \mathrm{U} / \mathrm{mL}$ & $\mathrm{p}$ Value for trend & & \\
\hline No. of subjects & 1969 & 1554 & 1529 & 1684 & & & \\
\hline $\mathrm{HAA}, \mathrm{cm}^{3}$, median (IQR) & $116.6(99.0$ to 142.5$)$ & 119.9 (101.7 to 143.0$)$ & 123.6 (102.9 to 148.1$)$ & 124.5 (105.2 to 149.2$)$ & & & \\
\hline \multicolumn{8}{|c|}{ Per cent difference $(95 \% \mathrm{Cl})$ in HAA } \\
\hline Unadjusted & 0 (Ref) & 1.99 (0.03 to 3.99$)$ & 5.15 (3.11 to 7.22$)$ & $6.26(4.25$ to 8.30$)$ & $<0.001$ & $1.65(1.10$ to 2.21$)$ & $<0.001$ \\
\hline Adjusted* & 0 (Ref) & 1.73 (0.15 to 3.33$)$ & 2.18 (0.57 to 3.82$)$ & 3.25 (1.65 to 4.88$)$ & $<0.001$ & $0.95(0.50$ to 1.40$)$ & $<0.001$ \\
\hline Never smoker & 0 (Ref) & $1.96(-0.37$ to 4.35$)$ & $1.02(-1.37$ to 3.46$)$ & 2.58 (0.12 to 5.09$)$ & 0.09 & $0.55(-0.17$ to 1.29$)$ & 0.14 \\
\hline \multirow[t]{2}{*}{ Ever smoker } & 0 (Ref) & $1.59(-0.53$ to 3.76$)$ & 3.46 (1.29 to 5.69$)$ & 4.35 (2.23 to 6.52$)$ & 0.001 & $1.35(0.78$ to 1.92$)$ & $<0.001$ \\
\hline & & & & & & $\begin{array}{l}\text { Prevalence ratio }(95 \% \mathrm{Cl}) \\
\text { for ILA per doubling of } \mathrm{RF} \text { IgA }\end{array}$ & \\
\hline No. of subjects & 747 & 617 & 516 & 523 & & & \\
\hline ILA prevalence, $\%(95 \% \mathrm{Cl})$ & $9.6(7.7$ to 12.0$)$ & $12.5(10.1$ to 15.3$)$ & 13.6 (10.9 to 16.8$)$ & 16.1 (13.2 to 19.5$)$ & & & \\
\hline \multicolumn{8}{|c|}{ Prevalence ratio $(95 \% \mathrm{Cl})$ for ILA } \\
\hline Unadjusted & 1 (Ref) & $1.29(0.96$ to 1.75$)$ & 1.41 (1.03 to 1.92$)$ & 1.67 (1.24 to 2.24$)$ & 0.001 & $1.15(1.08$ to 1.23$)$ & $<0.001$ \\
\hline Adjustedt & 1 (Ref) & $1.24(0.92$ to 1.68$)$ & $1.26(0.93$ to 1.71$)$ & $1.42(1.05$ to 1.91$)$ & 0.038 & 1.11 (1.03 to 1.20$)$ & 0.006 \\
\hline Never smoker & 1 (Ref) & $0.96(0.60$ to 1.51$)$ & 0.88 (0.55 to 1.40$)$ & 1.15 (0.73 to 1.82$)$ & 0.46 & 1.04 (0.88 to 1.23$)$ & 0.68 \\
\hline Ever smoker & 1 (Ref) & 1.49 (0.99 to 2.22$)$ & 1.67 (1.11 to 2.52$)$ & 1.62 (1.09 to 2.42$)$ & 0.001 & 1.14 (1.05 to 1.23$)$ & 0.001 \\
\hline
\end{tabular}

*Adjusted for age, sex, race, study site, BMI, waist circumference, pack-years of smoking, current smoking status, total volume of imaged lung, per cent emphysema and tube current.

tAdjusted for age, sex, race, pack-years of smoking and current smoking status.

p Value for interaction of RF IgA and smoking was 0.15 in the HAA analysis and 0.31 in the ILA analysis.

HAA, high attenuation areas; ILA, interstitial lung abnormalities; RF, rheumatoid factor.

Table 4 Association of anti-CCP with high attenuation areas and interstitial lung abnormalities on CT

\begin{tabular}{|c|c|c|c|c|c|c|c|}
\hline & \multicolumn{5}{|l|}{ Anti-CCP quartile } & \multirow{2}{*}{$\begin{array}{l}\text { Per cent difference }(95 \% \mathrm{Cl}) \text { in } \\
\text { HAA per doubling of anti-CCP }\end{array}$} & \multirow[b]{2}{*}{$\mathrm{p}$ Value } \\
\hline & $<0.17 \mathrm{U} / \mathrm{mL}$ & $0.17-0.2 \mathrm{U} / \mathrm{mL}$ & $0.21-0.5 \mathrm{U} / \mathrm{mL}$ & $>0.5 \mathrm{U} / \mathrm{mL}$ & $\mathrm{p}$ Value for trend & & \\
\hline No. of subjects & 1706 & 1696 & 1711 & 1615 & & & \\
\hline HAA, $\mathrm{cm}^{3}$, median (IQR) & 119.7 (100.7 to 144.0$)$ & 122.4 (101.8 to 147.2$)$ & $121.2(102.2$ to 145.8$)$ & 121.4 (101.8 to 145.7$)$ & & & \\
\hline \multicolumn{8}{|c|}{ Per cent difference $(95 \% \mathrm{Cl})$ in HAA } \\
\hline Unadjusted & 0 (Ref) & 2.54 (0.54 to 4.58$)$ & $1.31(-0.67$ to 3.32$)$ & $1.72(-0.29$ to 3.77$)$ & 0.42 & $0.13(-0.73$ to 0.99$)$ & 0.77 \\
\hline Adjusted* & 0 (Ref) & 2.34 (0.75 to 3.95$)$ & $1.33(-0.25$ to 2.93$)$ & 2.35 (0.60 to 4.13$)$ & 0.06 & $0.57(-0.12$ to 1.27$)$ & 0.11 \\
\hline Never smoker & 0 (Ref) & $1.17(-1.21$ to 3.61$)$ & $-0.73(-3.07$ to 1.67$)$ & $0.68(-1.94$ to 3.38$)$ & 0.70 & $-0.40(-1.55$ to 0.77$)$ & 0.50 \\
\hline \multirow[t]{2}{*}{ Ever smoker } & 0 (Ref) & $2.83(0.73$ to 4.99$)$ & $2.66(0.52$ to 4.85$)$ & $3.64(1.29$ to 6.05$)$ & 0.02 & $1.31(0.45$ to 2.18$)$ & 0.003 \\
\hline & & & & & & $\begin{array}{l}\text { Prevalence ratio }(95 \% \mathrm{Cl}) \text { for } \\
\text { ILA per doubling of anti-CCP }\end{array}$ & \\
\hline No. of subjects & 603 & 589 & 625 & 582 & & & \\
\hline ILA prevalence, \% (95\% Cl) & $14.3(11.7$ to 17.3$)$ & $12.1(9.7$ to 14.9$)$ & $11.4(9.1$ to 14.1$)$ & 12.5 (10.1 to 15.5$)$ & & & \\
\hline \multicolumn{8}{|c|}{ Prevalence ratio $(95 \% \mathrm{Cl})$ for ILA } \\
\hline Unadjusted & 1 (Ref) & 0.85 (0.63 to 1.13$)$ & 0.80 (0.59 to 1.07$)$ & 0.88 (0.66 to 1.18$)$ & 0.70 & $1.08(0.95$ to 1.23$)$ & 0.25 \\
\hline Adjustedt & 1 (Ref) & 0.88 (0.66 to 1.17$)$ & 0.81 (0.61 to 1.08 ) & $0.82(0.62$ to 1.10$)$ & 0.33 & 1.05 (0.91 to 1.20$)$ & 0.52 \\
\hline Never smoker & 1 (Ref) & $0.73(0.47$ to 1.14$)$ & 0.65 (0.41 to 1.003$)$ & 0.57 (0.35 to 0.92$)$ & 0.06 & 0.66 (0.37 to 1.19$)$ & 0.17 \\
\hline Ever smoker & 1 (Ref) & $0.96(0.66$ to 1.40$)$ & 0.93 (0.64 to 1.37$)$ & $1.02(0.70$ to 1.47$)$ & 0.81 & 1.13 (1.02 to 1.24$)$ & 0.02 \\
\hline
\end{tabular}

*Adjusted for age, sex, race, study site, BMI, waist circumference, pack-years of smoking, current smoking status, total volume of imaged lung, per cent emphysema and tube current.

†Adjusted for age, sex, race, pack-years of smoking and current smoking status.

$\mathrm{p}$ Value for interaction of anti-CCP and smoking was 0.02 in the HAA analysis and 0.01 in the ILA analysis.

$\vec{\circ}$ Anti-CCP, anti-cyclic citrullinated peptide antibody; HAA, high attenuation areas; ILA, interstitial lung abnormalities.

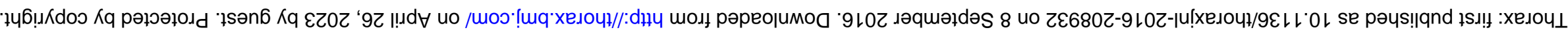



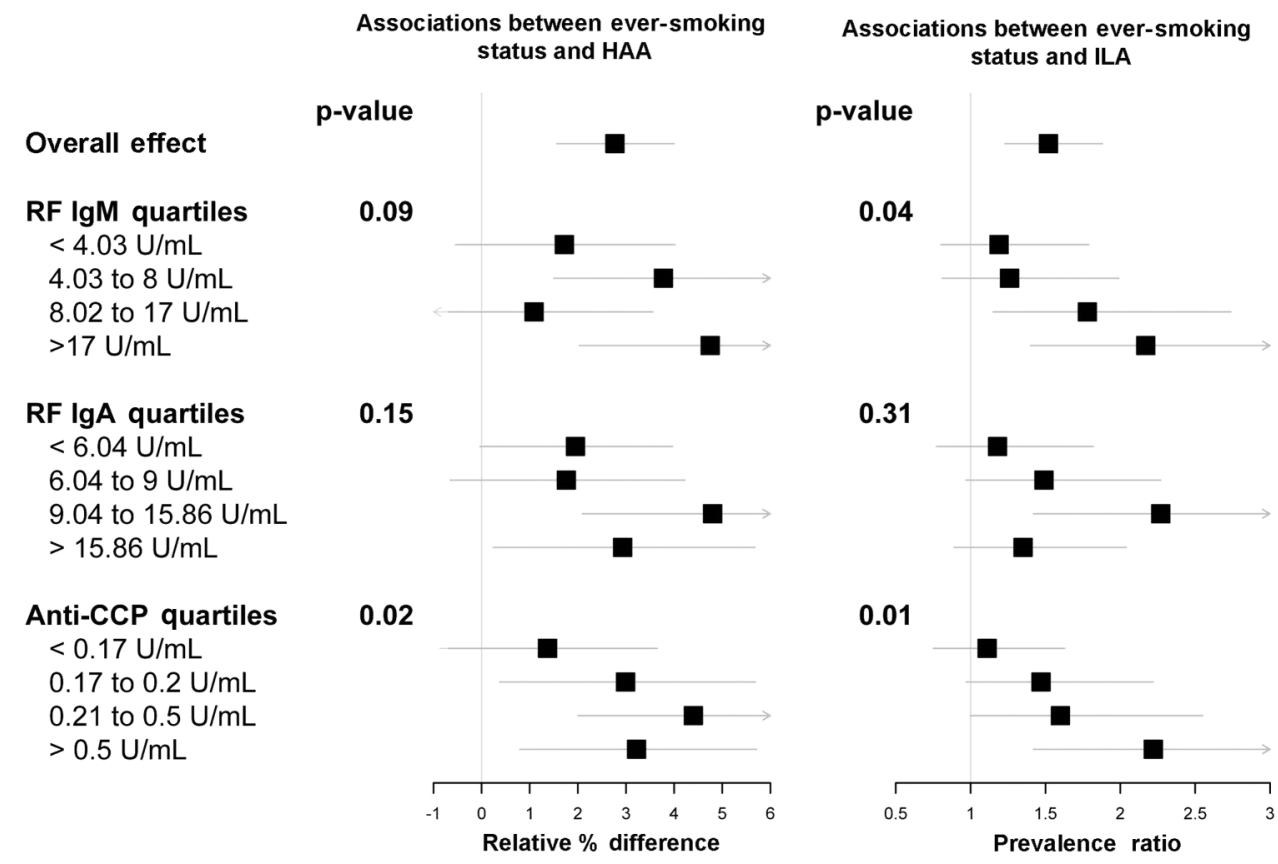

Figure 2 Forest plots showing associations between smoking status and high attenuation areas (left panel) and smoking status and interstitial lung abnormalities (ILAs) (right panel), stratified by quartiles of rheumatoid factor (RF) IgM, RF IgA and anti-cyclic citrullinated peptide antibody (anti-CCP). Boxes are point estimates. Horizontal lines are $95 \%$ Cls. p Values for interaction are shown.

absence of an established autoimmune disease. Our findings, therefore, suggest that higher serum RF levels, even in the absence of clinically evident RA, may contribute to lung injury and inflammation and to ILD risk. Future studies may show that RF levels aid in ILD risk stratification, even in the general population.

It has been well established that there is a preclinical phase of RA-related autoimmunity, during which circulating RF and/or anti-CCP are elevated without clinically apparent RA. ${ }^{25-28}$ Epitope spread targeting citrullinated proteins also occurs during this preclinical phase. ${ }^{29}$ The lung may be a target of this preclinical autoimmunity, or it may actually be the site of initiation of this preclinical autoimmunity-or both. ${ }^{22}$ Smoking is a well-established risk factor for the development of RA, for RF and anti-CCP positivity in RA, and for RF positivity in individuals without RA. ${ }^{30}{ }^{31}$ Smoking has been shown to increase levels of peptidylarginine deiminase 2 , an enzyme that catalyses the post-translational conversion of arginine to citrulline. ${ }^{32-34}$ An environmental exposure such as smoking could trigger the development of autoantigens in the lungs in response to inflammation, which may result in the local production of RF and anti-CCP. ${ }^{22}$ Our findings suggest that smoking may interact with RA-related autoimmunity to increase the risk of subclinical lung injury and inflammation in the general population, and also support the hypothesis that RA-related autoimmunity can be triggered by smoking-induced lung injury and inflammation. Future work should determine whether levels of antibodies to other citrullinated peptides and proteins, in addition to anti-CCP, are elevated in adults with subclinical ILD; whether adults with subclinical ILD have greater numbers of anticitrullinated peptide antibody reactivities compared with those without subclinical ILD; and whether epitope spread contributes to the development of ILA in the general population. ${ }^{35}$

Previous studies that have examined the association of different RF isotypes (ie, IgM and IgA) with RA disease manifestations and RA disease activity have had varying conclusions about the relevance of these isotypes. ${ }^{36} 37$ Although the presence of both RF IgM and RF IgA often predate the onset of RA by several years, and RF IgM is more readily identified in clinical testing, RF IgA has been shown to have greater sensitivity and positive predictive value than RF IgM for the diagnosis of RA and appears earlier in pre-disease sera. ${ }^{26}$ RF IgA, like RF IgM, has been associated with joint erosions in adults with RA. ${ }^{36}$ In a recent study of treatment-naïve adults with early RA, RF IgM, RF IgA and anti-CCP positivity were associated with erosive disease among ever smokers but not among never smokers. ${ }^{38}$ In our communitybased cohort, we found that smoking modified the associations of RF IgM and anti-CCP-but not RF IgA—with HAA and ILA. Our data suggest that RF IgA might lead to lung injury, inflammation and fibrosis in a smoking-independent fashion, while RF IgM and anti-CCP might require concomitant or prior cigarette smoking before ILD pathology develops.

There are some limitations of our study. First, HAA was ascertained on cardiac CT scans, which incompletely image the lungs. However, ILD preferentially affects the lower lobes of the lung, which were typically completely imaged, and numerous previous studies have demonstrated that MESA cardiac CT scans provide useful information about pulmonary emphysema. ${ }^{13} 17$ Second, we do not have data on whether any participants had clinical evidence of RA, although, given that the prevalence of $\mathrm{RA}$ in the US population is $1 \%$, it is likely that the majority of subjects were unaffected by RA. ${ }^{39} 40$ However, we cannot comment upon whether the associations between the autoantibodies we measured and ILA and HAA may be stronger among those with RA. Third, this is a cross-sectional study, so our ability to make causal inferences is limited. Although it is plausible that smoking interacts with RA-related autoantibodies to increase the risk of subclinical lung injury and inflammation in the general population, an alternative explanation may be that these RA-related autoantibodies are produced in response to smoking and lung inflammation. Finally, our study did not permit us to examine the mechanisms by which these autoantibodies might interact with smoking to contribute to subclinical lung injury and inflammation. Future studies should investigate these mechanisms. 
In summary, we have identified novel associations between RA-related autoantibodies and CT measures of subclinical lung injury, inflammation and ILD among community-dwelling adults, with stronger associations among ever smokers. Our findings support a potential role for autoimmunity in the development of subclinical ILD without regard to the presence of clinically evident autoimmune disease. Our study demonstrates that titres of RF and anti-CCP have meaning beyond a simple interpretation of 'positive' or 'negative'. The interaction between smoking and autoimmunity in the development of ILD deserves greater attention in future studies of ILD pathogenesis.

\author{
Author affiliations \\ 'Department of Medicine, Columbia University Medical Center, New York, \\ New York, USA \\ ${ }^{2}$ Department of Epidemiology, Columbia University Medical Center, New York, \\ New York, USA \\ ${ }^{3}$ Department of Radiology, Columbia University Medical Center, New York, \\ New York, USA \\ ${ }^{4}$ Department of Medicine and Center for Epidemiology and Biostatistics, University of \\ Pennsylvania Perelman School of Medicine, Philadelphia, Pennsylvania, USA \\ ${ }^{5}$ Department of Medicine, University of Washington, Seattle, Washington, USA \\ ${ }^{6}$ Department of Radiology, University of lowa Carver College of Medicine, lowa City, \\ Iowa, USA \\ ${ }^{7}$ Department of Radiology, University of Alabama at Birmingham School of \\ Medicine, Birmingham, Alabama, USA \\ ${ }^{8}$ Department of Medicine, Northwestern University Feinberg School of Medicine, \\ Chicago, Illinois, USA
}

\section{Twitter Follow David Lederer at @davidlederer}

Acknowledgements The authors thank the other investigators, the staff and the participants of the MESA study for their valuable contributions. A full list of participating MESA investigators and institutions can be found at http://www. mesa-nhlbi.org.

Contributors Substantial contributions to the conception or design of the work; or the acquisition, analysis or interpretation of data for the work: EJB, RGB, JHMA, SMK, GR, JLS, EAH, JDN, JRW, PHN, SKS, JMB, DSM, DJL. Drafting the work or revising it critically for important intellectual content: EJB, RGB, JHMA, SMK, GR, JLS, EAH, JDN, JRW, PHN, SKS, JMB, DSM, DJL. Final approval of the version submitted for publication: EJB, RGB, JHMA, SMK, GR, JLS, EAH, JDN, JRW, PHN, SKS, JMB, DSM, DJL. Accountability for all aspects of the work in ensuring that questions related to the accuracy or integrity of any part of the work are appropriately investigated and resolved: EJB, RGB, JHMA, SMK, GR, JLS, EAH, JDN, JRW, PHN, SKS, JMB, DSM, DJL.

Funding This research was supported by contracts N01-HC-95159, N01-HC-95160, N01-HC-95161, N01-HC-95162, N01-HC-95163, N01-HC-95164 and

N01-HC-95165 from the NHLBI and by grants UL1-TR-000040 and UL1-TR-001079 from NCRR. The MESA Lung Study is funded by R01-HL077612, RC1-HL100543 and R01-HL093081 from the NHLBI. The MESA Lung Fibrosis Study is funded by R01-HL103676 from the NHLBI and in part by a grant from the Pulmonary Fibrosis Foundation. The MESA Autoimmunity Study is funded by R01-HL104047 from the NHLBI and in part by an Arthritis Foundation Arthritis Investigator Award.

Competing interests DJL has received consulting fees from Genentech/Roche, Boehringer-Ingelheim, Gilead, Pharmakea, Veracyte, Patara Pharmaceuticals, Degge Group and the France Foundation related to IPF; Columbia University has received funding for clinical trials in IPF from Boehringer-Ingelheim, Gilead, Bayer, Global Blood Therapeutics and Fibrogen; Columbia University has received funding from the Pulmonary Fibrosis Foundation for DJL's consulting services; DJL has received fees for serving as a Deputy Editor for the Annals of the American Thoracic Society and as a Statistical Editor for Thorax. EAH is a founder and shareholder in VIDA Diagnostics. JDN is a consultant for VIDA Diagnostics and GSK; he is also a patent holder with and has stock options in VIDA Diagnostics. JRW is a member of the IPF Rally Educational Advisory Board and has received honoraria and travel expenses from the IPF Rally Educational Advisory Board.

Ethics approval MESA was approved by the Institutional Review Board for each participating study site.

Provenance and peer review Not commissioned; externally peer reviewed.

\section{REFERENCES}

1 Mittoo S, Gelber AC, Christopher-Stine L, et al. Ascertainment of collagen vascular disease in patients presenting with interstitial lung disease. Respir Med 2009;103:1152-8.
2 Travis WD, Costabel U, Hansell DM, et al. An official American Thoracic Society/ European Respiratory Society statement: update of the international multidisciplinary classification of the idiopathic interstitial pneumonias. Am J Respir Crit Care Med 2013;188:733-48.

3 Kinder BW, Collard HR, Koth L, et al. Idiopathic nonspecific interstitial pneumonia: lung manifestation of undifferentiated connective tissue disease? Am J Respir Crit Care Med 2007;176:691-7.

4 Sato T, Fujita J, Yamadori I, et al. Non-specific interstitial pneumonia; as the first clinical presentation of various collagen vascular disorders. Rheumatol Int 2006;26:551-5

5 Fischer A, Antoniou KM, Brown KK, et al. An official European Respiratory Society/ American Thoracic Society research statement: interstitial pneumonia with autoimmune features. Eur Respir J 2015:46:976-87.

6 Rosas 10, Ren P, Avila NA, et al. Early interstitial lung disease in familial pulmonary fibrosis. Am J Respir Crit Care Med 2007;176:698-705.

7 Washko GR, Hunninghake GM, Fernandez IE, et al. Lung volumes and emphysema in smokers with interstitial lung abnormalities. N Engl J Med 2011;364:897-906.

8 Gochuico BR, Avila NA, Chow CK, et al. Progressive preclinical interstitial lung disease in rheumatoid arthritis. Arch Intern Med 2008;168:159-66.

9 Podolanczuk AJ, Oelsner EC, Barr RG, et al. High attenuation areas on chest CT in community-dwelling adults: the MESA study. Eur Respir J 2016;48:1442-52.

10 Bild DE, Bluemke DA, Burke GL, et al. Multi-ethnic study of atherosclerosis: objectives and design. Am J Epidemiol 2002;156:871-81.

11 Majka DS, Vu TT, Pope RM, et al. Rheumatoid factors are associated with subclinical and clinical atherosclerosis in African American women: the Multi-Ethnic Study of Atherosclerosis (MESA). Arthritis Care Res (Hoboken). Published Online First: 9 May 2016. doi:10.1002/acr.22930

12 Lederer DJ, Enright PL, Kawut SM, et al. Cigarette smoking is associated with subclinical parenchymal lung disease: the Multi-Ethnic Study of Atherosclerosis (MESA)-lung study. Am J Respir Crit Care Med 2009;180:407-14.

13 Barr RG, Bluemke DA, Ahmed FS, et al. Percent emphysema, airflow obstruction, and impaired left ventricular filling. N Engl J Med 2010;362:217-27.

14 Hoffman EA, Jiang R, Baumhauer $\mathrm{H}$, et al. Reproducibility and validity of lung density measures from cardiac CT Scans-The Multi-Ethnic Study of Atherosclerosis (MESA) Lung Study. Acad Radiol 2009;16:689-99.

15 Guo J, Reinhardt JM, Kitaoka H, et al. Integrated system for CT-based assessment of parenchymal lung disease. Proceedings of the IEEE International Symposium on Biomedical Imaging, Washington DC, 7-10 July 2002; New York: Institution of Electrical and Electronics Engineers, 2002:871-4.

16 Hu S, Hoffman EA, Reinhardt JM. Automatic lung segmentation for accurate quantitation of volumetric X-ray CT images. IEEE Trans Med Imaging 2001;20:490-8

17 Oelsner EC, Hoffman EA, Folsom AR, et al. Association between emphysema-like lung on cardiac computed tomography and mortality in persons without airflow obstruction: a cohort study. Ann Intern Med 2014;161:863-73.

18 Hunninghake GM, Hatabu H, Okajima Y, et al. MUC5B promoter polymorphism and interstitial lung abnormalities. N Engl J Med 2013;368:2192-200.

19 Washko GR, Lynch DA, Matsuoka S, et al. Identification of early interstitial lung disease in smokers from the COPDGene Study. Acad Radiol 2010;17:48-53.

20 Rubin DB, Schenker N. Multiple imputation in health-care databases: an overview and some applications. Stat Med 1991;10:585-98.

21 Baumgartner KB, Samet JM, Stidley CA, et al. Cigarette smoking: a risk factor for idiopathic pulmonary fibrosis. Am J Respir Crit Care Med 1997;155:242-8.

22 Demoruelle MK, Weisman MH, Simonian PL, et al. Brief report: airways abnormalities and rheumatoid arthritis-related autoantibodies in subjects without arthritis: early injury or initiating site of autoimmunity? Arthritis Rheum 2012;64:1756-61.

23 Doyle TJ, Dellaripa PF, Batra K, et al. Functional impact of a spectrum of interstitial lung abnormalities in rheumatoid arthritis. Chest 2014;146:41-50.

24 Aubart F, Crestani B, Nicaise-Roland P, et al. High levels of anti-cyclic citrullinated peptide autoantibodies are associated with co-occurrence of pulmonary diseases with rheumatoid arthritis. J Rheumatol 2011;38:979-82.

25 del Puente A, Knowler WC, Pettitt DJ, et al. The incidence of rheumatoid arthritis is predicted by rheumatoid factor titer in a longitudinal population study. Arthritis Rheum 1988:31:1239-44.

26 Rantapaa-Dahlqvist S, de Jong BA, Berglin E, et al. Antibodies against cyclic citrullinated peptide and $\lg A$ rheumatoid factor predict the development of rheumatoid arthritis. Arthritis Rheum 2003:48:2741-9.

27 Majka DS, Deane KD, Parrish LA, et al. Duration of preclinical rheumatoid arthritis-related autoantibody positivity increases in subjects with older age at time of disease diagnosis. Ann Rheum Dis 2008;67:801-7.

28 Nielen MM, van Schaardenburg D, Reesink HW, et al. Specific autoantibodies precede the symptoms of rheumatoid arthritis: a study of serial measurements in blood donors. Arthritis Rheum 2004:50:380-6.

29 Sokolove J, Bromberg R, Deane KD, et al. Autoantibody epitope spreading in the pre-clinical phase predicts progression to rheumatoid arthritis. PLOS ONE 2012; 7:e35296

30 Jonsson T, Thorsteinsson J, Valdimarsson $\mathrm{H}$. Does smoking stimulate rheumatoid factor production in non-rheumatic individuals? APMIS 1998;106:970-4. 
31 Tuomi T, Heliovaara M, Palosuo T, et al. Smoking, lung function, and rheumatoid factors. Ann Rheum Dis 1990;49:753-6.

32 Kallberg $H$, Ding B, Padyukov L, et al. Smoking is a major preventable risk factor for rheumatoid arthritis: estimations of risks after various exposures to cigarette smoke. Ann Rheum Dis 2011;70:508-11.

33 Klareskog L, Stolt P, Lundberg K, et al. A new model for an etiology of rheumatoid arthritis: smoking may trigger HLA-DR (shared epitope)-restricted immune reactions to autoantigens modified by citrullination. Arthritis Rheum 2006:54:38-46.

34 Makrygiannakis D, Hermansson M, Ulfgren AK, et al. Smoking increases peptidylarginine deiminase 2 enzyme expression in human lungs and increases citrullination in BAL cells. Ann Rheum Dis 2008;67:1488-92.

35 Giles JT, Danoff SK, Sokolove J, et al. Association of fine specificity and repertoire expansion of anticitrullinated peptide antibodies with rheumatoid arthritis associated interstitial lung disease. Ann Rheum Dis 2014;73:1487-94.
36 Jonsson T, Thorsteinsson J, Kolbeinsson A, et al. Population study of the importance of rheumatoid factor isotypes in adults. Ann Rheum Dis 1992:51:863-8.

37 Eberhardt KB, Svensson B, Truedsson L, et al. The occurrence of rheumatoid factor isotypes in early definite rheumatoid arthritis—no relationship with erosions or disease activity. J Rheumatol 1988;15:1070-4.

38 Krol A, Garred P, Heegaard NH, et al. Interactions between smoking, increased serum levels of anti-CCP antibodies, rheumatoid factors, and erosive joint disease in patients with early, untreated rheumatoid arthritis. Scand I Rheumatol 2015;44:8-12.

39 Gabriel SE, Crowson CS, O'Fallon WM. The epidemiology of rheumatoid arthritis in Rochester, Minnesota, 1955-1985. Arthritis Rheum 1999;42:415-20.

40 Symmons DP. Epidemiology of rheumatoid arthritis: determinants of onset, persistence and outcome. Best Pract Res Clin Rheumatol 2002;16:707-22. 